\title{
Retrieval of pictures and words from long-term memory: Effects upon short-term memory
}

\author{
RICHARD V. KRANE, JAMES RANSOM, and JENNIFER L. MORRIS \\ Acadia University, Wolfville, Nova Scotia BOP IXO, Canada
}

\begin{abstract}
On each of a series of Brown-Peterson short-term memory (STM) tests, the presentation of a verbal to-be-remembered (TBR) item (a five-letter quintogram in Experiment 1 and an eightletter octogram in Experiment 2) was followed immediately by a 10-sec retention interval. During the retention interval, subjects recalled and reproduced previously learned paired associate response triads that consisted of three concrete pictures, concrete words, abstract pictures, or abstract words. Following selective retrieval of one of the four types of response triads from long-term memory (LTM), subjects attempted to recall the TBR items from STM. In both experiments, the intervening retrieval of abstract words resulted in significantly poorer retention of the TBR items than did the intervening retrieval of concrete words; the retrieval of concrete words resulted, in turn, in significantly poorer retention of the TBR items than did the retrieval of concrete or abstract pictures. Retrieval of the latter two types of materials appeared not to interfere with the short-term retention of the TBR verbal material (i.e., the quintograms and octograms). The results reflect the occurrence of selective interference effects, and, except for one discrepant finding, appear to be consistent with Paivio's (1971) dual-coding hypothesis and Shiffrin and Schneider's (1977) general theory of memory.
\end{abstract}

Shiffrin and Schneider's (1977) revision and extension of Atkinson and Shiffrin's (1968) multistore model of human memory conceptualizes memory as a large collection of nodes that become increasingly interassociated through learning. It is assumed that the majority of the nodes constitute the long-term store (LTS), a permanent passive repository for information, and that the nodes comprising LTS are normally in an inactive state. Short-term store (STS) is conceived as being a temporary state of activation of a subset of the nodes comprising LTS; that is, STS is a set of concurrently activated nodes in memory. It is further assumed that only a small amount of information will remain in an active state for several seconds or more, and that when the active nodes return to an inactive state, the information in STS is "lost" or forgotten. Of greater relevance to the present study is the assumption that "the rate of loss of any informational element or node in STS depends on the number of similar elements simultaneously active in STS. By similarity we refer not only to formal physical similarity but also to similarity of features at comparable levels of processing (e.g., the typeface of a printed word will be less likely than the word itself to cause forgetting of a verbally encoded second word in memory). When a large amount of similar information is present, the loss rate will be rapid but will slow as the amount of active information decreases" (Shiffrin \& Schneider, 1977, p. 157).

This research was reported at the annual meeting of the American Psychological Association in Washington, D.C., September 1976. Requests for reprints should be addressed to Richard V. Krane, Department of Psychology, Acadia University, Wolfville, Nova Scotia B0P 1X0, Canada.
Given the foregoing assumptions, one should expect the short-term retention of verbal material to vary as a function of the nature of the material presented during the retention interval of a Brown-Peterson (Brown, 1958; Peterson \& Peterson, 1959) short-term memory task. Distractor tasks requiring the processing of verbal information during the retention interval should increase the probability of to-be-remembered (TBR) verbal items' being "lost" or forgotten from STS. Although a number of studies have reported results consistent with this expectation, such studies have typically employed distractor tasks (e.g., shadowing) that require the processing of input emanating from some external source in the environment (Reitman, 1971, 1974; Shiffrin, 1973; Watkins, Watkins, Craik, \& Mazuryk, 1973). In terms of the Shiffrin and Schneider (1977) model, such tasks may be assumed to involve the automatic encoding of the sensory features of the distractor material, and the entry of that information into STS. That is, features of the distractor stimuli are presumed to be present in LTS, but inactive until automatically contacted by appropriate sensory input; the activation of a feature in LTS would be equivalent to placing the distractor stimuli in STS. However, in addition to such automatic encoding and deposition of information in STS, the STS is also presumed to receive selected input from the LTS. For example, the controlled transfer of information from LTS to STS might be assumed to occur in any situation in which information in LTS has some associative relationship to information currently being processed in STS, that is, situations involving problem solving, hypothesis testing, thinking, and so on. One of the purposes of the present 
study was to examine the retention of verbal information in STS as a function of the selective retrieval of different types of information from LTS during the retention interval of a Brown-Peterson short-term memory task. This was accomplished by employing a rehearsal-preventing distractor task that required the subject to recall and reproduce previously learned paired associate response terms that consisted of abstract words, concrete words, abstract pictures, or concrete pictures.

Recent tests of Paivio's (1971) dual-coding hy pothesis have suggested that concrete words and concrete pictures may be encoded and stored in both a verbalacoustic format and a visual-imaginal format, but that abstract words may be encoded and stored in only a verbal-acoustic format, and abstract pictures in only a visual-imaginal format (den Heyer \& Barrett, 1971; Kroll, Bee, \& Gurski, 1973; Murray \& Newman, 1973). Moreover, a series of experiments conducted by Pellegrino, Siegel, and Dhawan (1975, 1976a, 1976b), in which a Brown-Peterson paradigm was used to examine the short-term retention of words and pictures as a function of the nature of the distractor task (verbalacoustic or nonverbal-visual or both) performed during the retention interval, provides strong evidence of dual coding in short-term, as well as long-term, retention tasks. The pattern of selective interference effects obtained in the Pellegrino et al. experiments, as well as those obtained in other experiments (e.g., den Heyer \& Barrett, 1971; Kroll et al., 1973; Murray \& Newman, 1973; Nelson \& Brooks, 1973; Parkinson, 1972; Posner, 1967; Salzberg, Parks, Kroll, \& Parkinson, 1971) are consistent with the dual-coding hypothesis and suggest the operation of separate, independent acoustic and visual short-term memory systems.

\section{EXPERIMENT 1}

Given the assumption that the rate of loss of informational elements from the limited-capacity STS depends on the number of similar elements that are simultaneously active in STS (Shiffrin \& Schneider, 1977), and given the evidence suggesting the existence of a visualimaginal processing system that is independent of a verbal-acoustic processing system (Nelson \& Brooks, 1973; Pellegrino et al., 1975, 1976a, 1976b), a number of hypotheses can be generated concerning the effects of retrieving different types of materials (i.e., abstract and concrete pictures and words) from LTS upon the retention of verbal information in STS. For example, the short-term retention of a verbal TBR item (e.g., a quintogram comprised of five consonants) might be expected to be disrupted most by the retrieval from LTS of items presumably encoded in only a verbal-acoustic format (i.e., low-imagery abstract words), and to be disrupted least by the retrieval from LTS of items presumably encoded in only a visual-imaginal format (e.g., meaningless abstract shapes). Selective retrieval of abstract words from LTS should result in an increase in the number of similar (i.e., verbal) informational elements in STS, and hence, in a rapid rate of loss of the quintogram from STS, whereas the retrieval of abstract pictures should not.

In view of data reported by Nelson and Brooks (1973), which suggest that the verbal-acoustic characteristics and the visual-imaginal characteristics of high-imagery items are processed independently, one might also expect the retrieval of concrete pictures and concrete words to produce effects that are intermediate to those produced by the retrieval of abstract words and abstract pictures, with retention being more disrupted by the retrieval of concrete words than by the retrieval of concrete pictures. Thus, short-term retention of the quintogram might be expected to be best following the retrieval of abstract pictures, somewhat poorer, perhaps, following the retrieval of concrete pictures, poorer still following the retrieval of concrete words, and poorest following the retrieval of abstract words.

To investigate the effect of recalling these different types of materials from LTS upon the retention of verbal items (i.e., quintograms) in STS, the present experiments used a paired associate learning task in combination with a Brown-Peterson short-term memory paradigm. During the first phase of the experiment, each subject learned four different types of paired associates, the stimulus terms consisting of single digits and the response terms consisting of triads of concrete words, concrete pictures, abstract words, or abstract pictures. After each type of paired associate had been learned to a high criterion, and thus could be presumed to be stored in LTS, the second phase of the experiment was initiated. On each of a series of Brown-Peterson test trials, the presentation of a five-letter quintogram was followed immediately by the presentation of a single digit (i.e., one of the stimulus terms of the previously learned set of paired associates) that signaled the selective retrieval from LTS of the particular type of triad with which the digit had been paired during Phase 1. Following a brief interval, during which subjects recalled and reproduced the three concrete words, three concrete pictures, three abstract words, or three abstract pictures, the subjects attempted to recall the letters of the quintogram in correct serial order.

\footnotetext{
Method

Design and Subjects. The experiment involved two phases, a paired associate learning phase (Phase 1) and a Brown-Peterson test phase (Phase 2). The effects of imagery (abstract vs. concrete), mode of processing (pictures vs. words), and paired associate learning set (Set 1 vs. Set 2) upon paired associate learning and upon quintogram retention were investigated by means of a 2 by 2 by 2 repeated-measures factorial design Thirty-two Acadia University undergraduates served as volunteer subjects (16 males and 16 females).

Stimulus materials. The stimulus terms for the paired associates were single digits ranging from the number 1 to the number 8 , and the response terms were triads of concrete words, concrete pictures, abstract words, or abstract pictures. Items
} 
designated as concrete words were selected from the Paivio, Yuille, and Madigan (1968) list of nouns on the basis of their high imagery values (mean $=6.60)$. A set of simple black line drawings were used as concrete pictures. In order for a drawing to qualify as a concrete picture, 9 of 10 subjects in a pilot study had to respond with the same high-imagery word when asked to label the drawing. The mean imagery value of the words with which the subjects labeled the concrete pictures was equal to the mean imagery value for the concrete words (mean $=6.66$ ) Items chosen as abstract words were words rated low on the Paivio et al. $(1968)$ imagery scale $($ mean $=2.41)$. The items used as abstract pictures were black line drawings of four- to six-sided polygons selected from Vanderplas and Garvin's (1959) set of random shapes. The shapes were selected on the basis of a pilot study in which a procedure similar to that employed by Noble (1952) was used to index meaningfulness. The shapes selected were those that were found to elicit no meaningful associations and that subjects reported as being most difficult to label.

Thirty-six paired associate response triads were constructed such that any particular triad always consisted of three items of the same type (e.g., three abstract pictures). An attempt was also made to equate subjectively the associability of the items comprising a triad, and to minimize the probability of preexisting interitem associations. In view of the response requirements that would prevail during the $10-\mathrm{sec}$ retention interval of the Brown-Peterson tests in Phase 2, the various types of triads were constructed such that each type could be reproduced in 9-10 sec. The mean number of letters in the abstract word triads was 22.9 ; that for the concrete word triads was 23.1 .

Eight sets of four paired associates were prepared, each set containing each of the four types of paired associates. After each of two sets had been learned to criterion separately, the sets were combined to produce a list of eight paired associates. Thus, four different lists of paired associates were prepared, each list containing two sets (Set 1 and Set 2), and each set containing each of the four different types of paired associates.

Four additional paired associates were constructed, one for each of the four lists, by pairing the digit 9 with each of the four types of response triads. Each of the resulting paired associates was used equally of ten to provide subjects with a practice trial prior to learning the first set of paired associates.

The 32 quintograms ( 8 for each of the four lists of paired associates) that were used as the TBR items on the BrownPeterson test trials were constructed by randomly selecting 5 consonants, without replacement, from the 21 consonants of the alphabet; 4 additional quintograms were constructed for the practice trial associated with each of the four lists.

Apparatus. All paired associates were inscribed on $10 \times 15 \mathrm{~cm}$ white cards; numbers and letters (uppercase) were inscribed with Letraset Mini 1, and pictures were drawn in black ink, each picture being approximately $1.5 \mathrm{~cm}$ high. The cards on which the stimulus materials were inscribed were placed in a timercontrolled automatic card changer (Ralph Gerbrands Company, Model G1146), and presentation sequences were recorded and controlled by a Sony videotape recorder (Model AV-3600) and television monitor (Model CVM 192v). The height of the televised images of the numbers and letters was approximately $2.5 \mathrm{~cm}$, while that of the pictures was $5.0 \mathrm{~cm}$. Four videotapes, each of which was used to present one of the four lists of eight paired associates, were prepared for the paired associate learning phase, and one videotape, which contained the four different sets of eight Brown-Peterson test trials, was prepared for the test phase.

Procedure. Each subject was randomly assigned to learn one of the four lists of paired associates, with the restriction that an equal number of males and females be assigned to learn each list. Subjects were seated at a table $183 \mathrm{~cm}$ in front of the television monitor and were read instructions concerning the nature of the experiment and the sequence of events that would take place during the paired associate learning phase and the Brown-Peterson test phase.
Following a practice trial, which involved learning one of the four types of paired associates and being tested for quintogram retention on a single Brown-Peterson trial, each subject was required to learn each of the two sets of four paired associates constituting the assigned list. On each learning trial, the stimulus term (i.e., a single digit) of the paired associate was presented for $10 \mathrm{sec}$, a duration that had been determined by pilot research to be the minimum amount of time needed to recall and reproduce the picture or word triads. At the end of the $10 \mathrm{sec}$, a $1,000-\mathrm{Hz}$ tone was sounded that terminated the recall period and signaled the start of a 6 -sec study period, during which the stimulus and response triad were presented as a pair. Subjects first learned one set of four paired associates (Set 1) to a criterion of three consecutive correct trials, and then the second set (Set 2) to the same criterion (Criterion 1).

During the learning of each set, the order of presentation of the four paired associates was varied from trial to trial with the restriction that each of the four types be presented once in each block of four consecutive presentations. After attaining Criterion 1 on the second set, the subject was given the two sets of cards from which the videotape had been made and was allowed to review the combined set for about $5 \mathrm{~min}$. The combined list of eight paired associates was then presented via the videotape until the second criterion (Criterion 2) of three consecutive correct anticipation trials had been attained for each of the paired associates. Again, the order of presentation of the eight items comprising a list was varied with each list presentation.

Attainment of Criterion 2 was followed by the initiation of the Brown-Peterson short-term memory task. On each of the eight Brown-Peterson trials, a quintogram was presented for $2 \mathrm{sec}$ and was followed immediately by a $10-\mathrm{sec}$ presentation of the stimulus term of one of the paired associates learned in Phase 1. During the $10 \mathrm{sec}$, subjects were required to recall the triad of concrete pictures, concrete words, abstract pictures, or abstract words with which the stimulus had been associated and to reproduce the triad on the answer sheet provided by the experimenter. Following the $10-\mathrm{sec}$ recall-retention interval, an asterisk appeared on the television screen and a tone was sounded to signal recall of the quintogram. Subjects were allowed $10 \mathrm{sec}$ to write down the five letters of the quintogram in the same order in which they had appeared on the screen. After $10 \mathrm{sec}$ following the end of the quintogram recall period, another Brown-Peterson test trial was presented.

During the test phase, the order of presentation of the stimulus terms associated with the four types of response triads was counterbalanced across the four test lists to control for any possible proactive or retroactive inhibition effects that might occur as a function of the sequence of testing. Thus on Trial $n$ of the Brown-Peterson tests, each of the four test lists required a different type of response triad to be recalled prior to recall of the quintogram.

\section{Results and Discussion}

The data of primary interest are the number of letters of the quintogram that were recalled in correct serial order following recall of each of the four types of response triads of the paired associates in Set 1 and Set 2 . A 2 by 2 by 2 analysis of variance was conducted to determine the effects of mode of processing (pictures vs. words), imagery (concrete vs. abstract), and set membership (Set 1 vs. Set 2 ) of the paired associates upon the number of letters recalled from the quintogram. The results of the analysis indicated that retention of the quintogram was significantly poorer following the retrieval of word triads (i.e., concrete and abstract words) than it was following the retrieval of picture triads (i.e., concrete and abstract 
pictures) $[\mathrm{F}(1,31)=40.95, \quad \mathrm{MSe}=1.713, \mathrm{p}<.001]$. An average of only 3.28 letters $(66 \%)$ of the 5 letters of the quintogram were recalled following recall of the verbal material, while an average of 4.32 letters $(86 \%)$ were recalled following recall of the pictorial material. In contrast, retention of the quintogram was not found to be differentially affected by the retrieval of concrete triads (i.e., concrete pictures and words) and abstract triads (i.e., abstract pictures and words) $[\mathrm{F}(1,31)=1.76$, $\mathrm{MSe}=3.213, \mathrm{p}>.10 \mathrm{]}$; on the average, 3.65 letters (73\%) of the quintogram were recalled following recall of the abstract material, and 3.94 letters (79\%) were recalled following recall of the concrete material. Thus, manipulation of the mode of processing variable (pictures vs. words) exerted a greater differential effect upon the short-term retention of verbal material (i.e., the quintogram) than did manipulation of the imagery variable (abstractness vs. concreteness). Neither the main effect of set membership $(F<1)$ nor any of the firstorder or second-order interactions obtained significance (all Fs $<1.75, \mathrm{p}>.10$ ).

Given the conceptual framework of the present study, the differential retention effects observed among the four retrieval conditions are of particular interest. Figure 1 presents, both in terms of mean numbers of letters and in terms of mean proportions, the amount of the quintogram that was recalled correctly following the immediate prior recall of concrete pictures, abstract pictures, concrete words, and abstract words. In accord with the dual-coding hypothesis (Paivio, 1971) and the notion of separate processing systems for acoustic-verbal material and visual-imaginal material, planned pairwise

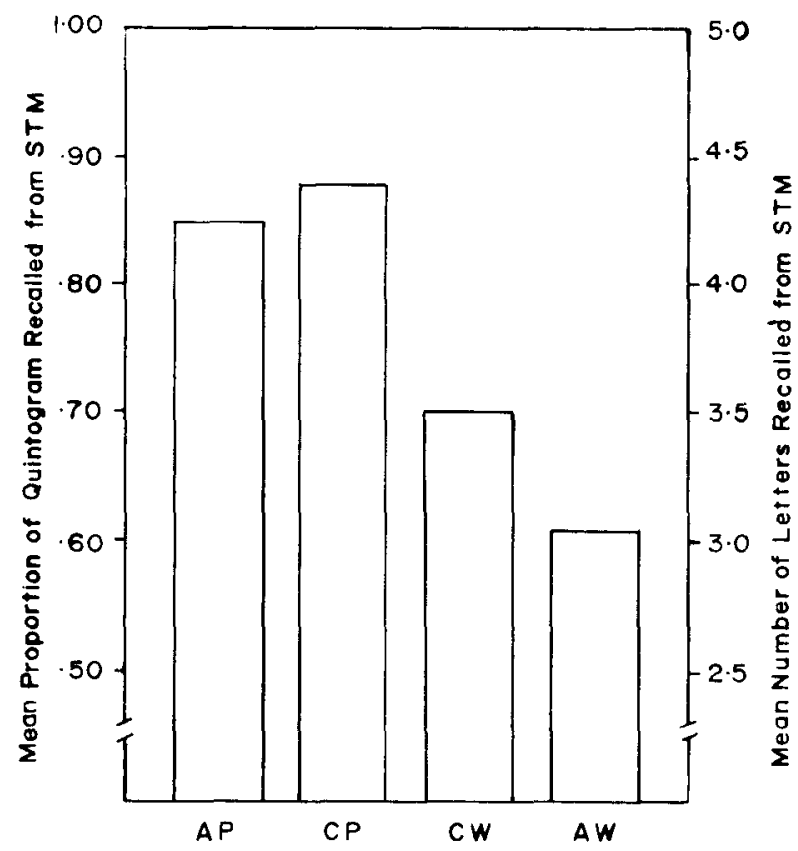

Type of Paired Associate Recalled from LTM

Figure 1. The effect of retrieving abstract or concrete pictures or words from long-term memory upon the retention of a quintogram in short-term memory. comparisons indicated that (1) the mean number of letters of the quintogram that were recalled following the retrieval of abstract words (mean $=3.05 ; 61 \%$ ) was significantly lower than the mean number recalled following the retrieval of concrete words (mean $=3.50$; $70 \%)[\mathrm{t}(31)=1.82, \mathrm{SE}=.254, \mathrm{p}<.05] ;$ (2) the mean number of letters recalled following the retrieval of concrete words was, in turn, significantly lower than the mean number recalled following the retrieval of concrete pictures (mean $=4.39 ; 88 \%$ ) $[\mathrm{t}(31)=3.56, \mathrm{SE}=.254$, $p<.01$ ] ; and (3) the mean number of letters recalled following the retrieval of abstract words was significantly lower than the number recalled following the retrieval of abstract pictures (mean $=4.25 ; 85 \%$ ) $[\mathrm{t}(31)=4.82, \quad \mathrm{SE}=.254, \quad \mathrm{p}<.01] . \quad$ Unanticipated, however, was the finding that retention of the quintogram was as good following the retrieval of concrete pictures as it was following the retrieval of abstract pictures $[\mathrm{t}(31)<1]$.

Except for the last finding, the results of the planned comparison are consistent with Paivio's (1971) dualcoding hypothesis and with Shiffrin and Schneider's (1977) conceptualization of the memory system, and they may be interpreted as providing support for these notions. In this regard, it may be noted that the observed differences in quintogram retention do not simply reflect differences in the associative strength (i.e., degree of learning) of the four types of paired associates. Table 1 presents the mean number of trials required to attain Criterion 1 and Criterion 2 of learning the paired associates. Inasmuch as the quintogram retention tests were not initiated until the criterion of learning had been attained the second time (Criterion 2), the mean numbers of trials required to attain Criterion 2 would seem to provide the more appropriate index of associative strength. That quintogram retention was not simply a function of the associative strength of the paired associates is evidenced by the lack of correspondence between the rank order of the four paired associate conditions on the Criterion 2 measure and their rank order on the quintogram retention measure. Whereas the mean number of trials required to attain Criterion 2 of learning was highest and virtually the same for the abstract picture and abstract word paired associates, and lowest for the concrete picture paired

Table 1

Mean Number of Trials Needed to Learn Each of the Four Types of Paired Associates to the First and Second Criteria of Three Successive Correct Recalls in Experiment 1

\begin{tabular}{llccc}
\hline & $\begin{array}{c}\text { Imagery } \\
\text { Level }\end{array}$ & Pictures & Words & Mean \\
\hline \multirow{3}{*}{ Criterion 1 } & Abstract & 7.14 & 7.64 & 7.39 \\
& Concrete & 5.98 & 7.08 & 6.53 \\
& Mean & 6.56 & 7.36 & \\
& Abstract & 4.09 & 4.10 & 4.09 \\
Criterion 2 & Concrete & 3.26 & 3.68 & 3.47 \\
& Mean & 3.67 & 3.89 & \\
\hline
\end{tabular}


associates, retention of the quintogram was highest and virtually the same in the abstract picture and concrete picture conditions, and lowest in the abstract word condition. In view of these incongruencies between the trials-to-Criterion 2 data and the quintogram retention data, it is rather unlikely that differences in the ease of accessing or retrieving the paired associates were responsible for the differences in quintogram retention.

Thus it seems reasonable to interpret the observed differences in quintogram retention as reflecting differences in the extent to which (1) the various types of paired associate response triads were encoded and stored in LTS in a verbal-acoustic format, a visualimaginal format, or both (Paivio, 1971); and (2) the controlled transfer of the various types of information from LTS to STS caused differential rates of loss of the quintogram from STS (Shiffrin \& Schneider, 1977). That the number of letters of the quintogram retained in short-term memory following the retrieval of concrete pictures was not less than that retained following the retrieval of abstract pictures was somewhat surprising. It was assumed that because concrete pictures readily elicit their corresponding verbal labels (Paivio, 1972; Paivio \& Csapo, 1969), some verbal-acoustic encoding would accompany the visual-imaginal encoding during the learning of the concrete picture paired associates, but not during the learning of the abstract picture paired associates. Thus, it was expected that the retrieval of concrete pictures would involve, at least to some extent, the operation of the verbal-acoustic processing system, whereas the retrieval of abstract pictures would involve the operation of the visual-imaginal system alone. This, in turn, was expected to result in the verbal elements of the quintogram suffering more interference in the former case than in the latter case. The fact that retention of the quintogram following the retrieval of concrete pictures was as high as that following the retrieval of abstract pictures suggests that subjects were able to selectively retrieve information that was coded and stored in a format that was different from the format in which the quintogram was coded and stored in STS. In other words, it appears that subjects may have been able to suppress or inhibit the retrieval of information that would interfere with the continued processing of the quintogram in STS when an alternative means of retrieving that information was both appropriate and economical.

However, an alternative explanation for the failure to find a difference in quintogram retention for the abstract picture and concrete picture retrieval conditions is suggested by the near-perfect retention that was observed in these two conditions. Inspection of each of the subject's scores revealed that 26 of the 32 subjects were able to recall all five letters of the quintogram on at least one of the two test trials involving the retrieval of concrete picture response triads, and that 27 of the 32 subjects were able to recall all five letters on at least one of the two test trials involving the retrieval of the abstract picture response triads. In view of these findings, a second experiment was conducted to determine whether or not the failure to obtain a reliable difference in quintogram retention for the concrete picture and abstract picture retrieval conditions was an artifact of the very high levels of performance observed under the two conditions, that is, a "ceiling effect."

\section{EXPERIMENT 2}

To overcome the problem of interpretation caused by the near-perfect retention of the quintograms in Experiment 1, Experiment 2 used octograms of eight consonants as the TBR items instead of quintograms. A supplementary purpose of Experiment 2 was to replicate the findings of the first experiment, but with new lists of paired associates. Although quintogram retention was not found to differ among the four different lists of paired associates used in Experiment 1, two new lists of paired associates were constructed so as to minimize any possibility of confounding that might occur as a function of uncontrolled differences in meaningfulness, word frequency, word length, or imagery.

\section{Method}

The method and procedures used in Experiment 2 were essentially the same as those used in Experiment 1, except for the following.

Subjects. Thirty-two graduate and undergraduate students enrolled in various summer programs at Acadia University served as subjects ( 12 males and 20 females).

Stimulus materials. Four sets of paired associates were constructed, each set containing the four different types of response triads. Two lists of eight paired associates each were then formed by combining two of the sets to form List 1 and two to form List 2. Thus, as in Experiment 1, each list contained two (one from Set 1 and one from Set 2) of each of the four types of paired associates.

The Paivio et al. (1968) list of nouns was again used to select a set of 12 concrete words and a set of 12 abstract words that, while differing in imagery [concrete words, mean $=6.53 ;$ abstract words, mean $=3.07 ; \mathrm{t}(22)=21.09$, $\mathrm{SE}=.164, \mathrm{p}<.001]$, did not differ reliably in meaningfulness (concrete words, mean $=6.39$; abstract words, mean $=6.00$ ), word frequency (concrete words, mean $=60.50$; abstract words, mean $=67.92$ ), or word length (concrete words, mean $=5.17$; abstract words, mean $=5.33$ ) (all ts $<1.88, \mathrm{p}>.05$, two-tailed). The 12 concrete pictures used to construct the response triads consisted of simple line drawings of the referent objects of the 12 concrete words (the concrete word triads of List 1 were used as concrete picture triads in List 2 , and vice versa), and thus they were identical to concrete words in terms of imagery, meaningfulness, word frequency, and word length values. The 12 abstract pictures were randomly selected from the set of 24 used in Experiment 1.

The eight octograms used as the TBR items in the BrownPeterson tests were constructed from the 21 consonants of the alphabet. No letter was allowed to occur twice in the same octogram, or in the same serial position in different octograms; in addition, no two letters were allowed to occur twice in the same serial order.

Eight different sequences of eight Brown-Peterson test trials 
were devised so as to permit a retention test of each of the eight octograms following the recall and reproduction of each type of response triad.

Procedure. The procedures used during the paired associate learning phase were exactly the same as those used in Experiment 1 . The Brown-Peterson test phase involved the presentation and recall of 10 octograms. Presentations of Octograms 1 and 10 were each followed by immediate recall of the octogram. These trials provided a measure of the extent to which an octogram could be recalled when recall was not delayed and the retrieval of other items from LTS was not required. The short-term retention tests for each of Octograms 2-9 involved exactly the same procedure as in Experiment 1 except that the octograms were presented for a period of $4 \mathrm{sec}$ instead of $2 \mathrm{sec}$.

\section{Results and Discussion}

Figure 2 presents the results of the Brown-Peterson short-term memory tests for retention of the octogram following the recall of each of the four types of response triads in Experiment 2. The first thing to note is that the use of octograms, rather than quintograms, eliminated the potential problem of ceiling effects. The mean number of letters recalled under any one of the four retrieval conditions did not exceed more than about half of the number of letters in the TBR octogram. The second point to note is that the pattern of results obtained across the four conditions conforms exactly to the pattern of results obtained in Experiment 1. A mixeddesign analysis of variance, with mode of processing (picture vs. words) and imagery (concrete vs. abstract) as within-subjects variables and lists (List 1 vs. List 2) as the between-subjects variable, indicated that retention of the octogram was significantly poorer following the retrieval of word triads as opposed to picture triads

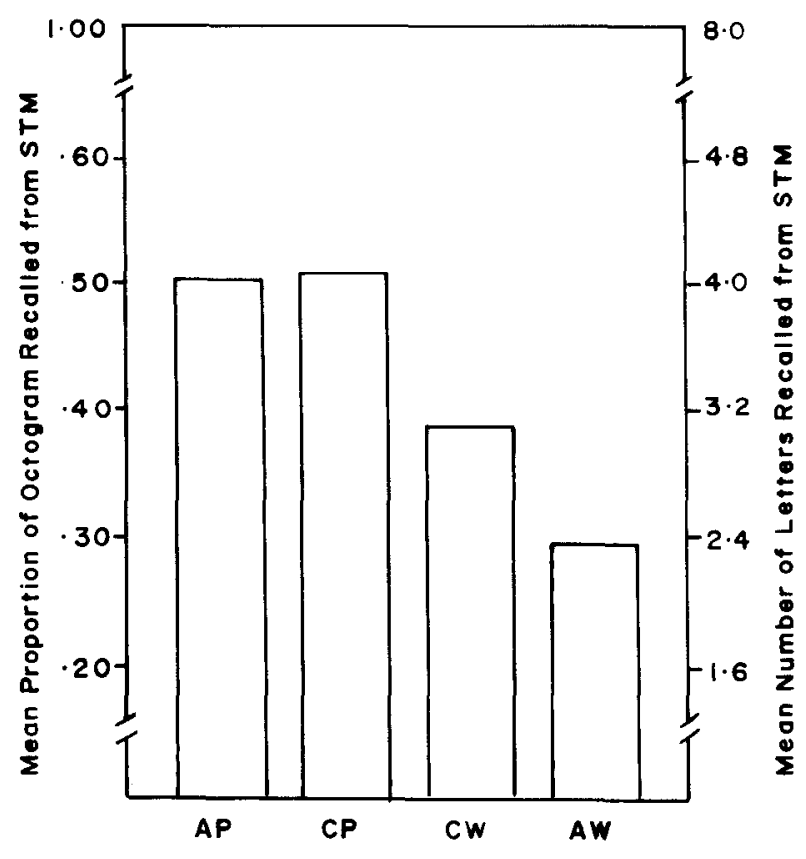

Type of Polred Associate Recalled from LTM

Figure 2. The effect of retrieving abstract or concrete pictures or words from long-term memory upon the retention of an octogram in short-term memory.
$[F(1,30)=28.65, \quad \mathrm{MSe}=3.76, \quad \mathrm{p}<.001] . \quad$ On the average, 4.05 letters $(51 \%)$ of the octogram could be recalled from STS following the recall of pictures, whereas only 2.75 letters (34\%) could be recalled following the recall of words. In contrast, the effect of retrieving abstract triads during the retention interval did not differ significantly from the effect of retrieving concrete triads during the interval $[\mathrm{F}(1,30)=2.85$, $\mathrm{MSe}=3.43, \mathrm{p}<.10]$; the recall of abstract triads resulted in an average of 3.20 letters (40\%) of the octogram being retained in STS, and the recall of concrete triads resulted in an average of 3.59 letters (45\%) being retained in STS. Short-term retention of the octogram also was found to not differ as a function of retrieving the triads from List 1 paired associates as opposed to List 2 paired associates [mean $=3.36$ letters $(42 \%)$ vs. mean $=3.44$ letters $(43 \%), F<1]$. While none of the first-order or second-order interactions was significant (all Fs $<1.74$, ps $>.10$ ), planned comparisons of the individual conditions indicated that retention of the octogram was significantly poorer following the retrieval of abstract words (mean $=2.39$ letters; $30 \%$ ) as compared to concrete words (mean $=3.11$ letters; $39 \%)[\mathrm{t}(30)=2.05, \mathrm{SE}=.351, \mathrm{p}<.025]$, and that the retrieval of concrete words, in turn, resulted in significantly poorer retention of the octogram than did the retrieval of concrete pictures (mean $=4.08$ letters; $51 \%)[\mathrm{t}(30)=2.76, \mathrm{SE}=.351, \mathrm{p}<.001]$. And, as was found in Experiment 1, the retrieval of concrete pictures did not disrupt retention of the octogram any more than did the retrieval of abstract pictures (mean $=4.02$ letters; $50 \%)(\mathrm{t}<1)$.

It is of interest to note that when recall of the octogram was not delayed by the retrieval of other information from LTS, as it was not for the octogram presentation that preceded the eight Brown-Peterson test trials and the octogram presentation that followed the test trials, subjects were able to recall an average of 4.22 letters (53\% retention) of Octogram 1 and 4.09 letters $(51 \%$ retention) of Octogram 10 . If the mean number of letters that could be recalled on these two immediate recall trials may be presumed to provide an estimate of the span of immediate memory, then it appears that the retrieval of either abstract pictures or concrete pictures caused no additional loss of the octogram from STS on the Brown-Peterson test trials. Whereas the mean number of letters that could be recalled following the retrieval of abstract pictures or concrete pictures did not differ reliably from the mean number that could be recalled on the two control trials (both ts $<1$ ), the mean number of letters that could be recalled following the retrieval of concrete words or abstract words was significantly lower than the number that could be recalled on the control trials $[\mathrm{t}(31)=3.20$, $\mathrm{SE}=.327, \quad \mathrm{p}<.01, \quad$ and $\mathrm{t}(31)=7.60, \quad \mathrm{SE}=.232$, $p<.001$, respectively]. These data suggest that the nearperfect retention of the quintogram following the retrieval of abstract or concrete pictures in Experiment 1 
Table 2

Mean Number of Trials Needed to Learn Each of the Four Types of Paired Associates to the First and Second Criteria of Three Successive Correct Recalls in Experiment 2

\begin{tabular}{llccc}
\hline & $\begin{array}{c}\text { Imagery } \\
\text { Level }\end{array}$ & Pictures & Words & Mean \\
\cline { 3 - 5 } Criterion 1 & Abstract & 7.37 & 5.03 & 6.20 \\
& Concrete & 4.66 & 5.53 & 5.10 \\
& Mean & 6.01 & 5.28 & \\
Criterion 2 & Abstract & 4.83 & 3.83 & 4.33 \\
& Concrete & 3.56 & 3.67 & 3.62 \\
& Mean & 4.20 & 3.75 & \\
\hline
\end{tabular}

was due to the fact that (1) the amount of information to be retrieved from STS (i.e., the information contained in the quintogram) did not exceed the span of immediate memory, and (2) the recall and reproduction of information encoded in a visual-imaginal format did not interfere with the retention of information encoded and stored in STS in a verbal-acoustic format.

Table 2 shows the mean number of trials required to learn each of the four types of paired associates to Criterion 1 and Criterion 2 during Phase 1 of the experiment. Again, as in Experiment 1, there is an apparent lack of correspondence between the rank orders of the four paired associate conditions on the Criterion 2 measure and their rank orders on the octogram retention measure. Although the number of trials required to attain Criterion 2 of learning was highest for the abstract picture paired associates and lowest for the concrete picture paired associates, retention of the octogram was highest and virtually the same in these two conditions. Conversely, despite the fact that subjects required essentially the same numbers of trials to attain Criterion 2 with the concrete picture and concrete word paired associates, retention of the octogram was significantly lower following the retrieval of concrete words as compared to concrete pictures. Thus, it is improbable that the pattern of results obtained on the Brown-Peterson short-term memory tests of octogram retention was due to differences in the associative strength or degree of learning of the different types of paired associates. Most convincing of this conclusion is that the pattern of results observed for the octogram retention tests in Experiment 2 was exactly the same as the pattern of results observed for the quintogram retention tests in Experiment 1, even though different patterns of results were obtained in Experiments 1 and 2 in terms of the numbers of trials required to learn the various types of paired associates to Criterion 1 and Criterion 2 of learning. The latter discrepancy may have been due to the elimination of differences in the meaningfulness, word frequency, or word length of the abstract word and concrete word response triads in Experiment 2.

\section{GENERAL DISCUSSION}

The results of the present experiments indicate that the selective retrieval of pictorial information from longterm memory has virtually no effect upon the short-term retention of abstract verbal information such as that contained in the TBR quintograms or octograms. This was found to be the case whether the pictorial information was meaningful and could be readily verbalized (i.e., concrete pictures) or it was meaningless and could not be readily verbalized (i.e., abstract pictures). In contrast, the selective retrieval of verbal information from long-term memory was found to have a decremental effect upon the short-term retention of the verbal TBR items, the magnitude of this effect being more pronounced when the retrieval from long-term memory involved abstract words (the referents of which were not readily imageable) than when it involved concrete words (the referents of which were highly imageable).

Although these results complement the results of numerous studies that have demonstrated a consistent superiority in the learning and retention of pictorial information (cf. Paivio, 1971), it would appear more appropriate to interpret them as reflecting the occurrence of selective interference effects, that is, effects similar to those observed in studies that have investigated the effects of processing visual-imaginal as opposed to verbal-acoustic information during the retention interval of a verbal memory task (e.g., Greenberg, 1977; Mendell, 1972; Pellegrino et al., 1975, 1976a, 1976b; Reitman, 1971, 1974; Salthouse, 1975; Shiffrin, 1973; Warren, 1977; Watkins et al., 1973). A selective interference interpretation would appear to be more appropriate for three reasons: First, such an interpretation would be consistent with the hypotheses generated on the basis of Shiffrin and Schneider's (1977) model of human memory and Paivio's (1971) dual-coding hypothesis. Second, the differential effects of retrieving the various types of information from long-term memory were found not to be systematically related to differences in the rates of learning the information (as indicated by the trials-to-Criterion 1 data) or to differences in the accessibility of the information (as indicated by the trials-to-Criterion 2 data). The third reason rests on the assumption that the TBR quintograms and octograms were encoded and stored in short-term memory primarily in a verbal-acoustic format, ${ }^{1}$ and that, as a consequence, the number of attributes held in common with the TBR items was greatest for the abstract words, somewhat less for the concrete words, and least for the concrete and abstract pictures. Given this assumption, the amount of information retained in short-term memory appeared to vary as a function of the degree of similarity (i.e., the number of attributes assumed to be 
held in common) between the information retrieved from long-term memory and the information to be retained in short-term memory; the greater the degree of similarity, the poorer the retention of the TBR items.

That the retrieval of concrete pictures caused as little forgetting of the TBR items as did the retrieval of abstract pictures was an unexpected finding. On the basis of Paivio's (1971) dual-coding hypothesis, it was assumed that, in contrast to abstract pictures, the retrieval of concrete pictures would result in the activation of a verbal-acoustic representation as well as a visual-imaginal representation, and that activation of the verbal-acoustic representation would interfere with retention of the TBR items. This assumption now appears untenable: In addition to finding that the TBR items were recalled equally well following the retrieval of either concrete pictures or abstract pictures, it was found that the amount recalled under these conditions did not differ from the amount recalled immediately following presentation of the TBR item, that is, in the absence of having to retrieve information from longterm memory. Thus, one might presume that neither the retrieval of abstract pictures nor the retrieval of concrete pictures resulted in the activation of a verbal-acoustic representation.

Although retention of the TBR items was not differentially affected by the retrieval of abstract and concrete pictures, it was differentially affected by the retrieval of abstract and concrete words, and in a manner consistent with the dual-coding hypothesis. It is of interest to note, however, that the asymmetrical effects of retrieving the two types of words and pictures were not consistent with Paivio's (1975) more recent assumption of transfer asymmetry. According to this assumption, the transfer of information from the visual-imaginal system to the verbal-acoustic system occurs more readily than does the converse. Given this assumption, one might have expected the retrieval of concrete pictures to result in the activation of a verbal-acoustic representation more readily than the retrieval of concrete words would result in the activation of a visual-imaginal representation. Thus, one might have been led to predict that retention of the TBR items would be differentially affected by the retrieval of abstract and concrete pictures significantly more than it would be differentially affected by the retrieval of abstract and concrete words. That the observed asymmetry was directly opposite to that which might have been predicted on the basis of the transfer asymmetry assumption suggests a need for determining the boundary conditions of the assumption. It may be that transfer of information from the visual-imaginal system to the verbal-acoustic system occurs more rapidly than does the converse during encoding and storage operations, but more slowly during "decoding" and retrieval operations.

\section{REFERENCES}

Atkinson, R. C., \& Shifrrin, R. M. Human memory: A proposed system and its control processes. In K. W. Spence \& J. T. Spence (Eds.), The psychology of learning and motivation: Advances in research and theory (Vol. 2). New York: Academic Press, 1968.

Brown, J. Some tests of the decay theory of immediate memory. Quarterly Journal of Experimental Psychology, 1958, 10, 12-21.

DEN HeYer, K., \& BARRETT, B. Selective loss of visual and verbal information in STM by means of visual and verbal interpolated tasks. Psychonomic Science, 1971, 25, 100-102.

Greenberg, S. N. Competition between memory and perceptual tasks involving physically similar stimuli. American Journal of Psychology, 1977, 90, 675-687.

Kroll, N. E. A., BeE, J., \& Gurski, G. Release of proactive interference as a result of changing presentation modality. Journal of Experimental Psychology, 1973, 98, 131:137.

MENDELL, P. R. Short-term visual memory: Comparative effects of two types of distraction on the recall of visually presented verbal and nonverbal material. Journal of Experimental Psychology, 1972, 94, 244-247.

Murray, D. J., \& Newman, F. M. Visual and verbal coding in short-term memory. Journal of Experimental Psychology, 1973, 100, 58-62.

Nelson, D. L., \& Brooks, D. H. Independence of phonetic and imaginal features. Journal of Experimental Psychology, 1973, 97, 1-7.

Noble, C. E. An analysis of memory. Psychological Review, 1952, 59, 421-430.

Paivio, A. Imagery and verbal processes. New York: Holt, Rinehart, \& Winston, 1971

Paivio, A. A theoretical analysis of the role of imagery in learning and memory. In P. W. Sheean (Ed.), The function and nature of imagery. New York: Academic Press, 1972.

Paivio, A. Perceptual comparisons through the mind's eye. Memory \& Cognition, 1975, 3, 635-647.

Paivio, A., \& Csapo, K. Concrete image and verbal memory codes. Journal of Experimental Psychology, 1969, 80, 279-285.

Paivio, A., Yuille, J. C., \& Madigan, S. A. Concreteness, imagery, and meaningfulness. Journal of Experimental Psychology Monograph, 1968, 76(1, Part 2).

Parkinson, S. R. Short-term memory while shadowing: Multipleitem recall of visually and of aurally presented letters. Journal of Experimental Psychology, 1972, 92, 256-265.

Pellegrino, J. W., Siegel, A. W., \& Dhawan, M. Short-term retention of pictures and words: Evidence for dual coding systems. Journal of Experimental Psychology: Human Learning and Memory, 1975, 104, 95-102.

Pellegrino, J. W., Siegel, A. W., \& Dhawan, M. Differential distraction effects in short-term and long-term retention of pictures and words. Journal of Experimental Psychology: Human Learning and Memory, 1976, 2, 541-547. (a)

Pellegrino, J. W., Siegel, A. W., \& Dhawan, M. Shortterm retention of pictures and words as a function of type of distraction and length of delay interval. Memory \& Cognition, 1976, 4, 11-15. (b)

Peterson, L. R., \& Peterson, M. J. Short-term retention of individual items. Journal of Experimental Psychology, 1959, 58, 193-198.

Posner, M. I. Characteristics of visual and kinesthetic memory codes. Journal of Experimental Psychology, 1967, 75, 103-107.

REITMAN, J. S. Mechanisms of forgetting in short-term memory. Cognitive Psychology, 1972, 2, 185-195.

Reitman, J. S. Without surreptitious rehearsal, information in short-term memory decays. Journal of Verbal Learning and Verbal Behavior, 1974, 13, 365-367. 
Salthouse, T. A. Simultaneous processing of verbal and spatial information. Memory \& Cognition, 1975, 3, 221-225.

Salzberg, P. M., Parks, T. E., Kroll, N. E. A., \& Parkinson, S. R. Retroactive effects of phonemic similarity on short-term recall of visual and auditory stimuli. Journal of Experimental Psychology, 1971, 91, 43-46.

Shiffrin, R. M. Information persistence in short-term memory. Journal of Experimental Psychology, 1973, 100, 39-49.

Shiffrin, R. M., \& Schneider, W. Controlled and automatic human infurmation processing: II. Perceptual learning, automatic attending, and a general theory. Psychological Review, 1977, 84, 127-196.

VANDERPLAS, J. M., \& Garvin, E. A. The association value of random shapes. Journal of Experimental Psychology, 1959, 57, 147-154.
Warren, M. W. The effects of recall-concurrent visual-motor distraction on picture and word recall. Memory \& Cognition, $1977,5,362-370$.

Watkins, M. J., Watkins, O. C., Craik, F. I. M., \& Mazuryk, G. Effect of nonverbal distraction on short-term storage. Journal of Experimental Psychology, 1973, 101, 296-300.

\section{NOTE}

1. One should be mindful of the possibility that the BrownPeterson procedure (especially for eight-item strings) might have involved a long-term component as well.

(Received for publication July 20, 1978; revision accepted March 15, 1979.) 